\title{
Incidence risk of PD-I/PD-LI related diarrhea in non-small cell lung cancer (NSCLC) patients: a systematic review and meta-analysis
}

This article was published in the following Dove Press journal: Cancer Management and Research

\author{
Caiqing Zhang ${ }^{1} *$ \\ Shuisheng Zhang ${ }^{2, *}$ \\ Deguo $\mathrm{Xu}^{3, *}$ \\ Rujun Liu ${ }^{4, *}$ \\ Qingshan Zhu ${ }^{5}$ \\ Yi Zhao ${ }^{6}$ \\ Yantao $\mathrm{Mao}^{7}$ \\ Yuan $\operatorname{Tian}^{3}$
}

'Department of Respiratory, Shandong Provincial Qianfoshan Hospital Affiliated to Shandong University, Jinan, Shandong 2500 I4, People's Republic of China;

${ }^{2}$ Department of General Surgery, Peking University Third Hospital, Beijing I00191, People's Republic of China; ${ }^{3}$ Department of Radiotherapy Oncology, Shandong Provincial Qianfoshan Hospital Affiliated to Shandong University, Jinan, Shandong 2500 I4, People's Republic of China; ${ }^{4}$ Department of Oncology, LongKou People's Hospital, Yantai, Shandong 26570I, People's Republic of China; ${ }^{5}$ Department of Radiotherapy oncology, Anyang Cancer Hospital of Henan Province, Anyang, Henan 455000,

People's Republic of China; ${ }^{6}$ Department of Oncology, The First Affiliated Hospital of Dalian Medical University, Dalian, Liaoning II6000, People's Republic of China; ${ }^{7}$ Department of Oncology, Yantaishan Hospital of Shandong Province, Yantai, 264000, People's Republic of China

*These authors contributed equally to this work

Correspondence: Yuan Tian

Department of Radiotherapy Oncology, Shandong Provincial Qianfoshan Hospital, NO. 16766, Jingshi Road, LiXia District, Jinan, Shandong 2500 I4, People's Republic China

Tel +86 I3954197326

Email tytytianyuan@aliyun.com
Purpose: We designed the study to illustrate the OR of programmed cell death-1 (PD-1) or ligand 1 (PD-L1) inhibitor-related diarrhea in patients with non-small cell lung cancer.

Method: This systematic review and meta-analysis were put into practice according to the Preferred Reporting Items for Systematic Reviews and Meta-analyses (PRISMA) guidelines. Incidence of all grades for PD-1/PD-L1 inhibitor-related diarrhea in NSCLC was taken into account.

Results: After screening and eligibility assessment of 57 articles, a total of 12 clinical trials involving 6,659 participants were collected for the final meta-analysis. The incidence risk of diarrhea for all grades was lower in PD-1 inhibitor monotherapy compared to monochemotherapy of docetaxel $\left(\mathrm{OR}=0.31,95 \% \mathrm{CI}[0.24,0.41] ; \mathrm{I}^{2}=0 \%, \mathrm{Z}=8.23(p<0.00001)\right)$, while a similar result could also be seen in PD-L1 inhibitor monotherapy group (OR $=0.41,95 \% \mathrm{CI}$ $\left.[0.27,0.64] ; \mathrm{I}^{2}=59 \%, \mathrm{Z}=3.92[p<0.00001]\right)$. The opposite result can be seen when PD-1/PDL1 inhibitor combined chemotherapy was compared to chemotherapy alone (OR=1.51, 95\% CI $\left.[1.22,1.87] ; \mathrm{I}^{2}=0 \%, \mathrm{Z}=3.77[p<0.00001]\right)$. Similar incidence trend could also be seen in the meta-analysis of diarrhea for grade 1-2 and grade 3-5.

Conclusion: The incidence risk of diarrhea associated with PD-1/-PD-L1 inhibitor monotherapy was significantly lower than that of docetaxel monotherapy group. However it was higher in PD-1/PD-L1 inhibitor combined with chemotherapy group compared with the chemotherapy alone group.

Keywords: diarrhea, PD-1/PD-L1, NSCLC, meta-analysis

\section{Introduction}

The programmed cell death 1 (PD-1) receptor has emerged as a dominant negative regulator of antitumor T-cell effector function when engaged by its ligand programmed cell death ligand 1 (PD-L1), expressed on the surface of cells within a tumor. ${ }^{1}$ Therapies that target the PD-1 receptor have shown unprecedented rates of durable clinical responses in patients with various cancer types. One mechanism by which cancer tissues limit the host immune response is via playing a key role in the maintenance of immunological tolerance to self-antigens, preventing autoimmune disorders, while immune checkpoint inhibitors (ICIs), including CTLA-4 and PD-1, were able to unleash $\mathrm{T}$ cells to fight cancer. $^{2-6}$ Nivolumab showed its antitumor efficacy in non-small cell lung cancer (NSCLC) when it was first administered to a patient in October 2006 in a Phase I trial. ${ }^{7}$ With the development of clinical research, more and more PD-1/PD-L1 inhibitors have been tried in 
clinical trials for antilung cancer treatment and have shown good efficacy, ${ }^{8-10}$ especially for NSCLC. ${ }^{11-22}$

The toxic effects associated with PD-1/PD-L1 inhibitors may affect any organ and result from the activation of autoreactive $\mathrm{T}$ cells, thereby damaging the host tissue and even jeopardizing the patient's life. ${ }^{11-23}$ Diarrhea is a common side effect of antitumor medications. Moderate or severe diarrhea can cause electrolyte imbalance in patients, further leading to the interruption of antitumor treatment. ${ }^{11-22}$ There was no significant difference in the incidence of diarrhea between PD-L1 inhibitors and placebo. ${ }^{8}$ Compared with other antilung cancer treatment programs, there is no systematic analysis and report on the incidence risk of PD-1/PD-L1-related diarrhea. In order to clarify the incidence risk of diarrhea in the treatment of NSCLC with PD-1/PD-L1 inhibitors, we conducted a systematic review and meta-analysis.

\section{Methods}

This systematic review and meta-analysis was put into practice according to the Preferred Reporting Items for Systematic Reviews and Meta-analyses (PRISMA) guidelines. $^{24}$

\section{Types of studies}

We paid our attention mainly to randomized clinical trials, especially for Phase III clinical trials related to NSCLC. The criteria for the selected data: 1) PD-1/PD-L1-chemotherapy combinations compared with chemotherapy alone; 2) PD-1/ PD-L1 monotherapy compared with chemotherapy alone; 3 ) PD-1/PD-L1 plus antitumor therapy compared with PD-1/ PD-L1 inhibitor alone; 4) safety or toxicity was for evaluating OR with $95 \% \mathrm{CI}$ or other evaluable indicators such as $\mathrm{RR}, \mathrm{HR}$ and so on; and 5) subjects were diagnosed with NSCLC but SCLC. Some studies without useful information, such as observational studies, editorials, commentaries, and review articles, were excluded.

\section{Outcome and exposure of interest}

The outcome of interest was incidence rate of PD-1/PD-L1 inhibitor-related diarrhea, including any grade of diarrhea. Diarrhea-related death was considered to be secondary outcome. Study data that reported measures of colitis, including colitis-related diarrhea, was not taken into account. We sought to examine the difference of incidence risk between PD-1/PD-L1 group and control group.

\section{Search strategy and review method}

Original articles, related to results of prospective clinical trials of PD1/PD-L1 inhibitor regimens for NSCLC patients, including monotherapy and combination therapy, were verified by a Pubmed search. The date range was limited from January 22, 2013, to January 22, 2019. Keywords were displayed just as the following: "PD1/PD-L1", "nivolumab", "BMS-963558", "pembrolizumab/MK-3475", “atezolizumab/MPDL3280A", "Avelumab", "Durvalumab", "lung cancer", "NSCLC", "SCLC", "safety", and "toxicity".

We selected studies limited in human beings which were shown in full text, abstract, or poster form. Two members of our team were asked to identify their eligibility independently. References from review articles, editorials, and included studies were reviewed and cross-referenced to check completeness. If no useful data about toxicities of related drugs were found, we would try to get in touch with the corresponding author for more information, or it was precluded from the meta-analysis. The characteristics of enrolled studies, including first author, year of publication, drug name, treatment regimen, study design, phase, number of patients, number of PD-1/PD-L1-related diarrhea and baseline demographic characteristics, were extracted. Risk of bias was evaluated by the Cochrane Collaboration tool for assessing the risk of bias in randomized trials. ${ }^{25}$

\section{Assessment of heterogeneity and statistical analysis}

We took Newcastle-Ottawa scale, proposed by the Cochrane Collaboration, ${ }^{26}$ to check the quality of enrolled studies. Cochrane's Q statistic and the $\mathrm{I}^{2}$ statistic were used for accessing the heterogeneity among studies just as proposed by Higgins et al, ${ }^{27}$ while Harbord's test was taken to check publication bias for all studies. OR value was considered to be a much more conservative and sensitive evaluation parameter and might be more inclined to reveal a safety signal, as the method by which an OR is calculated provided a point estimate farther from unity than that provided by an HR. OR, and $95 \% \mathrm{CI}$ would be calculated by random effect (RE) for the heterogeneity inherent in the data.. ${ }^{28} p<0.05$ was deemed to be statistically significant for all the results of meta-analysis. Statistical tests were all two-sided. Metaanalysis was performed using Review Manager, version 5.3.

\section{Subgroup analysis}

We divided all the data into three groups according to the degree of diarrhea, including all grades of diarrhea, grade 1-2, 
and grade 3-5. We performed a number of subgroup analyses to assess the potential association between PD-1/PD-L1 inhibitor and chemotherapy in the incidence risk of diarrhea. We considered subgroups, including drug type (PD-1 or PD-L1 inhibitor), combination with chemotherapy (combination with chemotherapy or mono-therapy), and drug name.

\section{Results}

\section{Literature search results}

Among all the citations identified by our electronic and manual searches, 124 articles met the preliminary inclusion criteria. After screening and eligibility assessment of 57 articles, a total of 12 clinical trials involving 6,659 participants were collected for the final meta-analysis. ${ }^{11-22}$ The study flow diagram was shown in Figure 1. All studies included a subgroup analysis that compared the intervention group with the control group, with an OR for incidence risk of diarrhea (Figure 3-5).

\section{Characteristics of identified trials}

The main characteristics of all enrolled 12 studies are summarized in (Table 1). In total, 6,659 patients were included, of which 1,537 (23.1\%) experienced diarrhea. Study types included 9 Phase III studies; ${ }^{11-16,18,21,22} 1$ Phase II/III; ${ }^{20}$ and 2 Phase II; ${ }^{17,19}$ All included studies had a control chemotherapy group. 5 of enrolled NSCLC studies received previous therapy before PD-1/ PD-L1 inhibitor. ${ }^{11,16,17,20-22}$ The drug used in 4 studies was PD-L1 inhibitor, ${ }^{11,12,16,17}$ while the PD-1 inhibitor was used in the other 8 studies. ${ }^{13-15,18-22} 11$ studies had the results of diarrhea for grade $3-5 .^{11-16,18-22}$ In 6 studies, the drug used in the control group was monotherapy with docetaxel. ${ }^{11,16,17,20-22}$ In the other 6 trials, the control treatment regimen was combined chemotherapy. $^{12-15,18,19}$

\section{Risk of bias}

We used Newcastle-Ottawa scale to evaluate study quality and risk of bias in both comparison and noncomparison studies. All selected clinical studies were randomized and double-blinded. They were of high quality and at a low risk for selection bias. The risk of bias graph and summary are summarized in Figure 2A and B. The risk for performance and detection bias was too low to affect the outcome. Harbord's test statistic did not suggest obvious publication bias in funnel plot (Figure S1-S3).

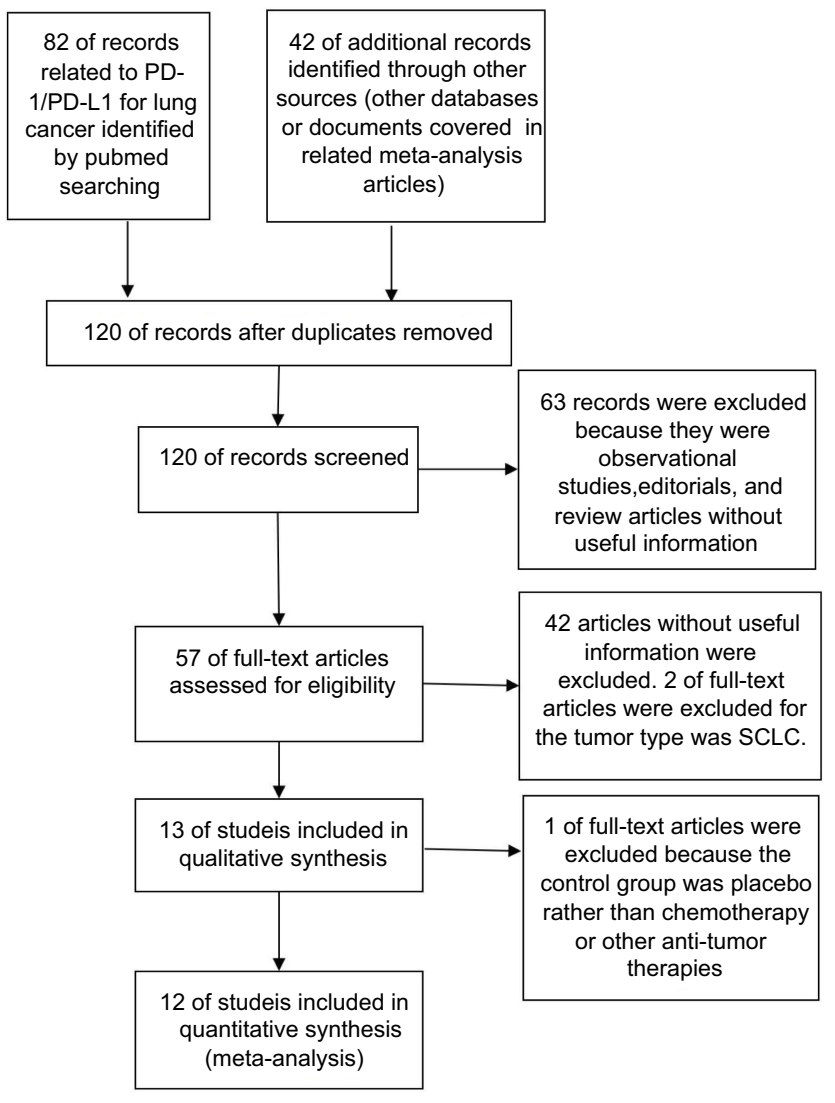

Figure I Study flow diagram of inclusion.

Abbreviations: PD-I, programmed cell death I; PD-LI, Programmed death-ligand I; SCLC, small cell lung cancer; NSCLC, non-small cell lung cancer.

\section{OR of diarrhea for all grades and grade I-2}

The data of 12 studies involving NSCLC patients with PD1/PD-L1 inhibitors data taken to make further analysis for diarrhea by grade. ${ }^{1-22}$ Diarrhea of all grades was taken to be evaluated first. All the enrolled studies were divided into 2 groups according to the treatment regimen. The treatment regimen of the experimental group was PD-1/ PD-L1 inhibitor monotherapy, named group $\mathrm{A},{ }^{11,13,16-}$ 18,20-22 while the treatment regimen of the experimental group was PD-1/PD-L1 inhibitor combined with chemotherapy, named group B. ${ }^{12,14,15,19}$

The overall outcome of meta-analysis for group A is summarized at the bottom of (Figure $3 \mathrm{~A})(\mathrm{OR}=0.45,95 \% \mathrm{CI}[0.31$, $\left.0.66], \mathrm{I}^{2}=79 \%, \mathrm{Z}=4.17[p<0.00001]\right),{ }^{11,13,16-18,20-22}$ while the opposite result of group $\mathrm{B}$ is displayed in Figure $3 \mathrm{~B}(\mathrm{OR}=1.51$, $95 \%$ CI $\left.[1.22,1.87], \mathrm{I}^{2}=0 \%, \mathrm{Z}=3.77[p=0.0002]\right) .{ }^{12,14,15,19}$ Funnel plot is provided in Figure S1. The OR of PD-1 subgroup was a little higher $(\mathrm{OR}=0.48,95 \% \mathrm{CI}[0.27,0.86])$ than that of PD-L1 subgroup (OR=0.41, 95\% CI [0.27, 0.64]) (Figure 3A1). Due to the existence of heterogeneity, we 


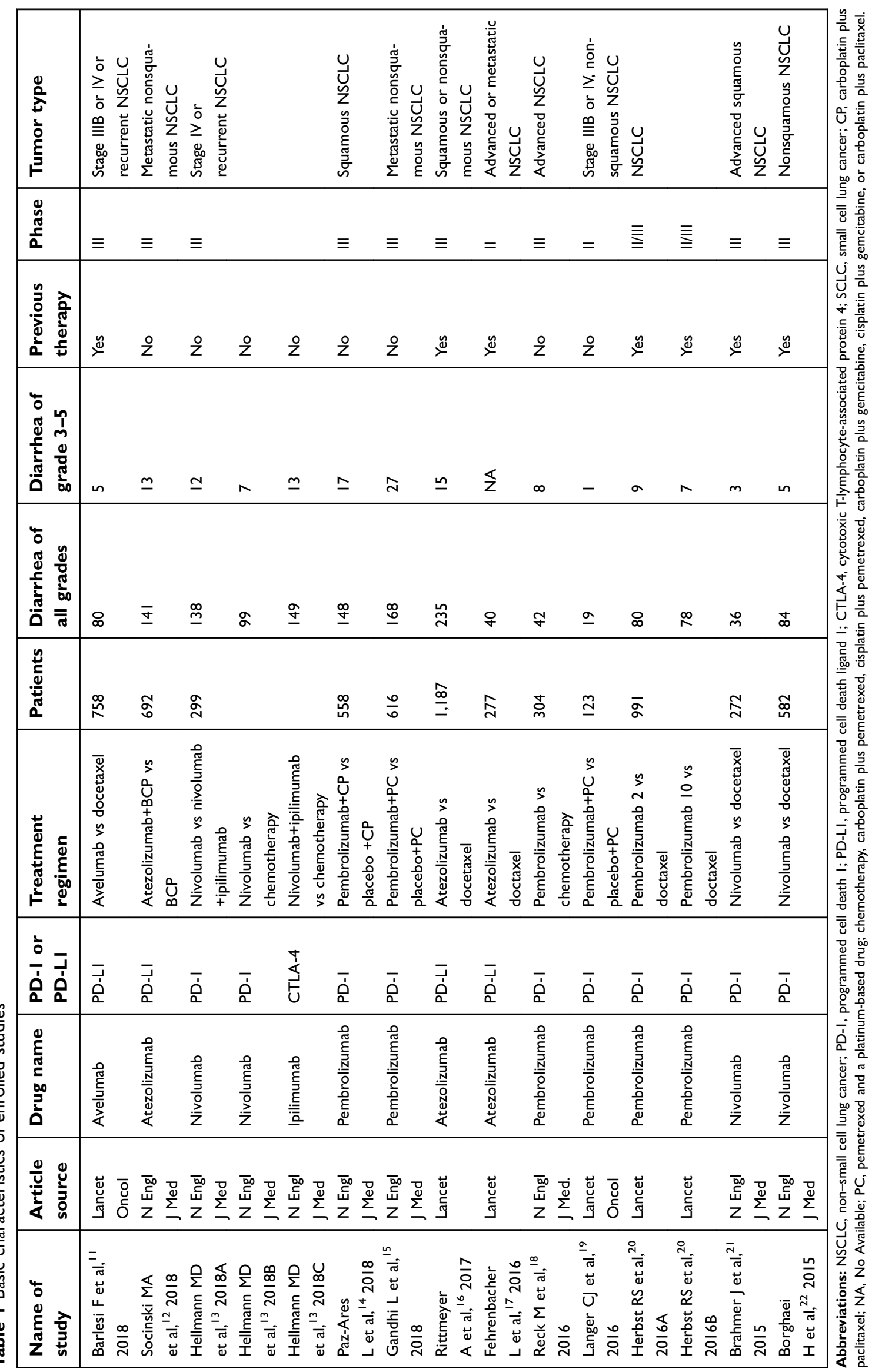



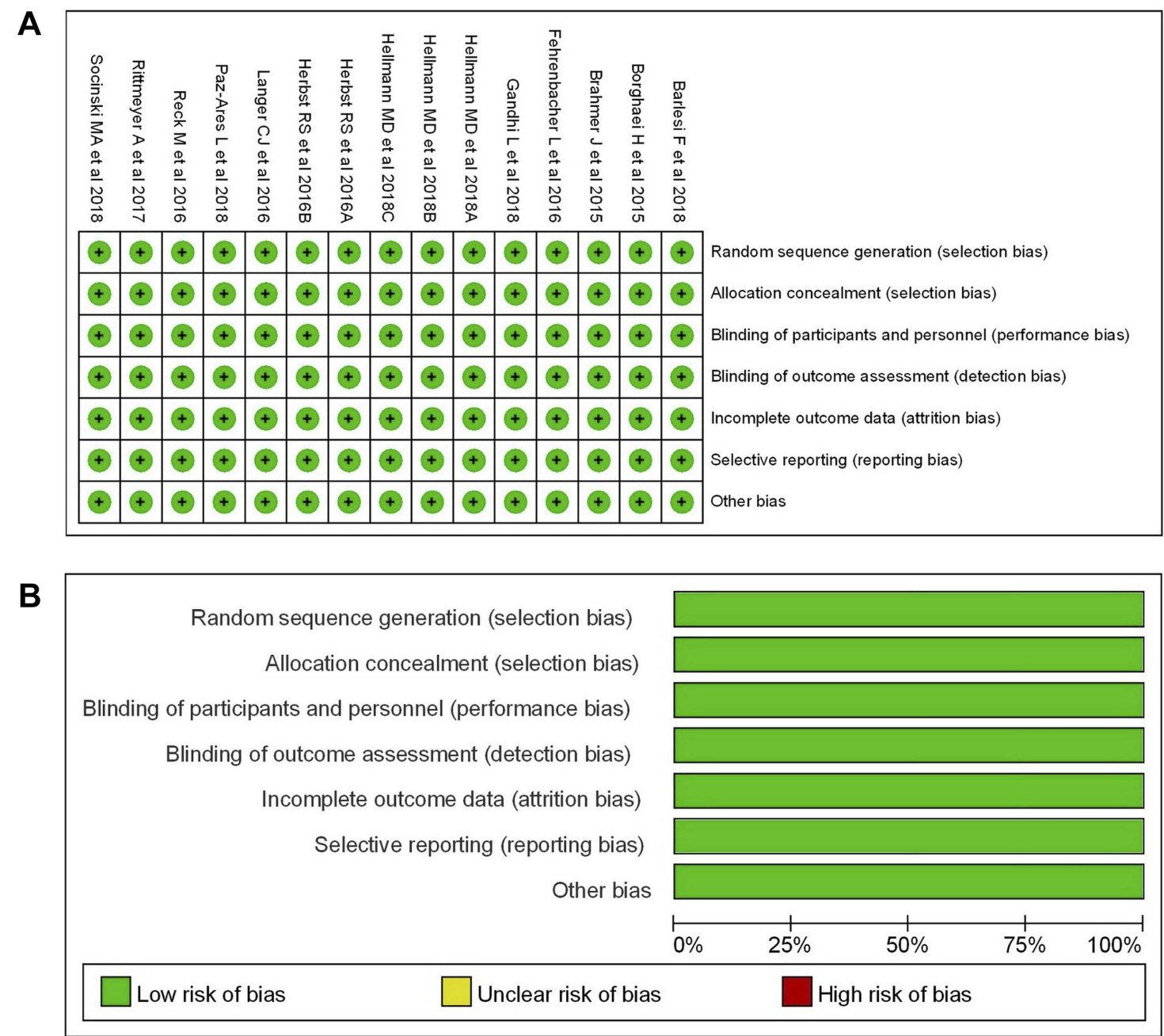

Figure 2 (A): Risk of bias summary: review authors' judgments about each risk of bias item for each included study. (B): Risk of bias graph: review authors' judgments about each risk of bias item presented as percentages across all included studies.

conducted a further subgroup analysis $\left(\mathrm{I}^{2}=79 \%\right)$, three different subgroup analysis methods were used for group A (Figure 3A1-A3). ${ }^{11,13,16-18,20-22}$

Regardless of whether the experimental group was single-agent PD-1 or PD-L1 inhibitor, the incidence risk of subgroup meta-analysis for diarrhea was significantly lower than that of the control group related to docetaxel $\left(\mathrm{OR}=0.31,95 \% \mathrm{CI}[0.24,0.41], \mathrm{I}^{2}=0 \% ; \mathrm{OR}=0.41,95 \% \mathrm{CI}\right.$ $[0.27,0.64], \mathrm{I}^{2}=59 \%$; Figure $\left.3 \mathrm{~A} 2\right)$. The results of the two subgroup meta-analysis were statistically significant. Heterogeneity was only found in the subgroup of PD-L1 inhibitor $\left(\mathrm{I}^{2}=59 \%\right.$, Figure $\left.3 \mathrm{~A} 2\right) .{ }^{11,16,17}$ However, the similar trend could not be seen when PD-1/PD-L1 inhibitor was compared with combination chemotherapy $(\mathrm{OR}=1.16$, $95 \%$ CI $[0.81,1.64], \mathrm{I}^{2}=0 \%$; Figure $\left.3 \mathrm{~A} 2\right) .{ }^{13,18}$

When another subgroup analysis was performed based on the specific drug name of the PD-1/PD-L1 inhibitor, compared with the control group, it was found that except for nivolumab, the incidence of diarrhea in the PD-1/PD-L1 group was lower than that of the control group, and the results were statistically significant (Figure 3A3). In the subgroup of nivolumab $\left(\mathrm{I}^{2}=91 \%\right)$ and atezolizumab $\left(\mathrm{I}^{2}=70 \%\right)$, there was significant heterogeneity in the analysis results (Figure $3 \mathrm{~A} 3$ ) .

After subgroup analysis of group B data, we found that PD-1 inhibitor combined with chemotherapy significantly increased the incidence risk of diarrhea, and the results were statistically significant ( $\mathrm{OR}=1.55,95 \%$ CI $[1.19,2.02]$, $\mathrm{I}^{2}=0 \%, \mathrm{Z}=3.24$ [ $\left.p=0.001\right]$; Figure 3B). ${ }^{14,15,19}$

Then, the incidence rates of grade 1-2 diarrhea were taken into account. The incidence risk of diarrhea for all grades (Figure 3) and grade 1-2 (Figure 4) were almost in the same trend. The details of the meta-analysis for grade 1-2 related to group A are summarized in (Figure 4A1-3) $\left(\mathrm{OR}=0.50, \quad 95 \% \quad\right.$ CI $\quad[0.35, \quad 0.71], \quad \mathrm{I}^{2}=75 \% ; \quad \mathrm{Z}=3.86$ 


\section{A1}

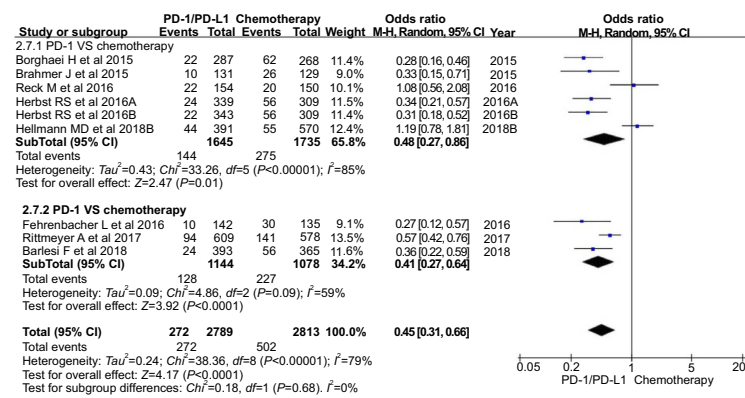

A2

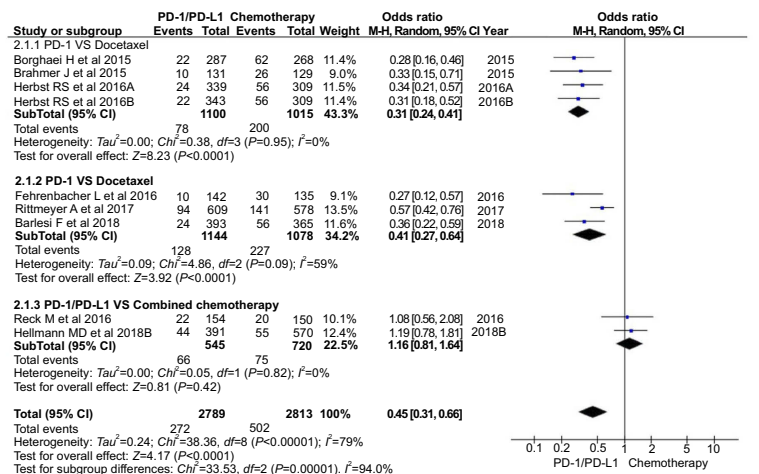

A3

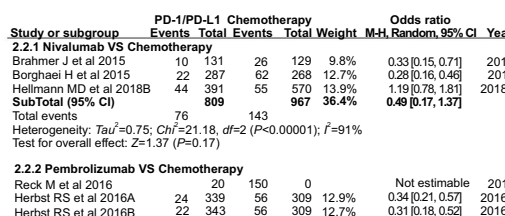

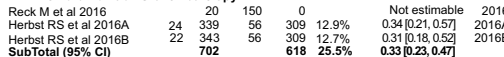

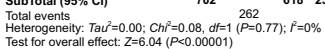

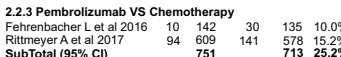

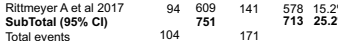

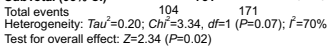

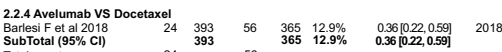

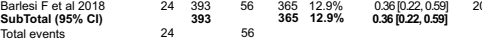

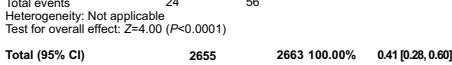

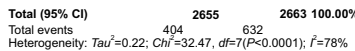

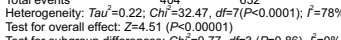
$0.41[028,0.00]$

B

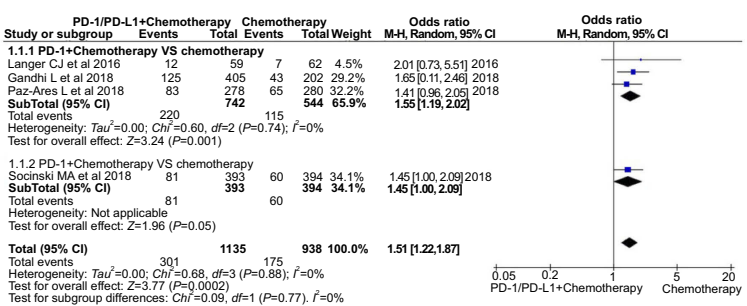

Figure 3 Forest plots of the incidence risk for all-grade diarrhea. (AI): OR of diarrhea for all grades in the subgroup analysis (PD-I/PD-LI vs chemotherapy). (A2): OR of diarrhea for all grades in the subgroup analysis (PD-I/PD-LI vs docetaxel/combined chemotherapy), the data included were assigned to the corresponding subgroup according to control group (docetaxel or combined chemotherapy). (A3): OR of diarrhea for all grades in the subgroup analysis (PD-I/PD-LI vs chemotherapy), the data included were assigned to the corresponding subgroup according to the name of PD-I/PD-LI inhibitor and the control group. (B): OR of diarrhea for all grades in the subgroup analysis (PD-I/PD-LI + chemotherapy vs chemotherapy).

Abbreviation: RE, random effect; PD-I, programmed cell death I; PD-LI, programmed cell death ligand I;.

[ $p=0.0001])$, while the opposite result of group $\mathrm{B}$ is displayed in Figure 4B $\left(\mathrm{OR}=1.39,95 \%\right.$ CI $[1.11,1.74], \mathrm{I}^{2}=0 \%, \mathrm{Z}=2.87$ $[p=0.004])$. The OR of PD-1 subgroup was a little lower $(\mathrm{OR}=0.49,95 \% \mathrm{CI}[0.29,0.84])$ than that of PD-L1 subgroup $(\mathrm{OR}=0.52,95 \% \mathrm{CI}[0.35,0.75])$ in (Figure 4A1). Regardless of whether the experimental group was single-agent PD-1 or PD-L1 inhibitor, the incidence risk of subgroup meta-analysis for diarrhea related to grade 1-2 was significantly lower than that of the control group related to docetaxel $(\mathrm{OR}=0.34,95 \%$ CI $[0.25,0.45], \mathrm{I}^{2}=0 \% ; \mathrm{OR}=0.52,95 \%$ CI $[0.35,0.75]$, $\mathrm{I}^{2}=44 \%$; Figure 4A2). When the subgroup analysis was performed based on the specific drug name of the PD-1/PD-L1 inhibitor, compared with the control group, it was found that except for nivolumab, the incidence rate of diarrhea in the PD1/PD-L1 group was lower than that of the control group, and the results were statistically significant (Figure 4A3). Funnel plot is provided in Figure S2.

\section{OR of diarrhea for grade 3-5}

The incidence of diarrhea related to grade 3-5 was reported in 11 studies. ${ }^{11-16,18-22}$ The results of the meta-analysis were gathered at the bottom of Figure 5. Different from the above results of all grades and grade 1-2, no statistical significance could be found when we put group A (PD-1/PD-L1 VS chemotherapy) into meta-analysis for the incidence risk of diarrhea $\left(\mathrm{OR}=0.45,95 \% \mathrm{CI}[0.20,1.04], \mathrm{I}^{2}=39 \%, \mathrm{Z}=1.88\right.$ $[p=0.06]$; Figure 5A). ${ }^{11,13,16,18,20-22}$ The incidence risk of diarrhea in the PD-1/PD-L1 combined chemotherapy group was higher than that in the control group, and the results were statistically significant $(\mathrm{OR}=2.07,95 \%$ CI $[1.12,3.82]$, $\mathrm{I}^{2}=1 \%, \mathrm{Z}=2.32[p=0.02]$; Figure $\left.5 \mathrm{~B}\right) .{ }^{12,14,15,19}$ Funnel plot is provided in Figure $\mathrm{S} 3$.

As the existence of heterogeneity $\left(\mathrm{I}^{2}=39 \%\right)$ in group A, stratified subgroup analysis was taken into account (Figure 5A1-A3). ${ }^{11,13,16,18,20-22}$ When the PD-1 inhibitor was compared with docetaxel (Figure 5A2), the incidence of diarrhea for grade 3-5 was significantly lower than that of the control group (OR=0.26, 95\% CI [0.09, 0.73], $\left.\mathrm{I}^{2}=0 \%, \mathrm{Z}=2.55[p=0.01]\right){ }^{20-22}$ The same trend of results can also be seen when the PD-L1 inhibitor was compared to docetaxel $\left(\mathrm{OR}=0.28,95 \%\right.$ CI $[0.10,0.82], \mathrm{I}^{2}=0 \%$, $\mathrm{Z}=2.33[p=0.02]$; Figure 5A2). ${ }^{11,16}$ When we mixed the single-agent docetaxel and the combination chemotherapy together as a control group, we did not find statistically significant results in the subgroup analysis (PD-1 vs chemotherapy) $\left(\mathrm{OR}=0.55,95 \% \mathrm{CI}[0.19,1.59], \mathrm{I}^{2}=46 \%\right.$, 
A1

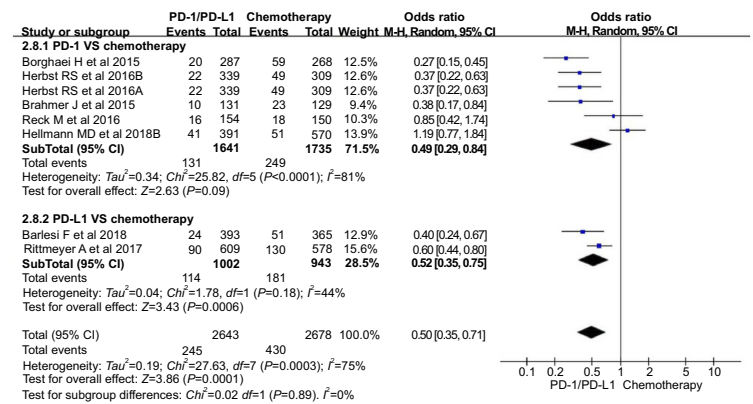

A2

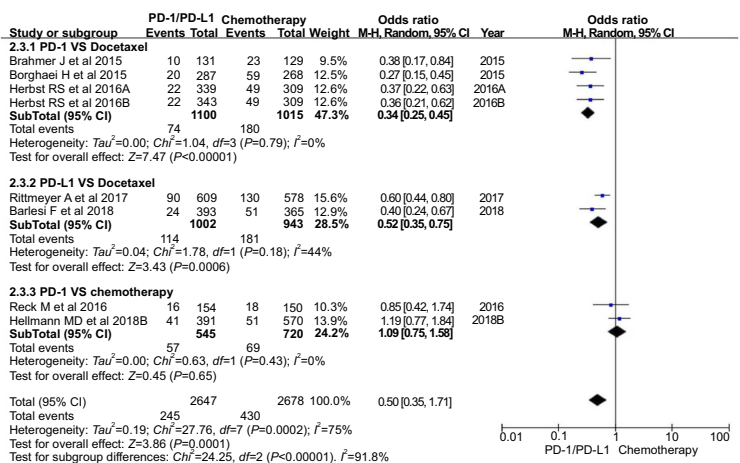

A3

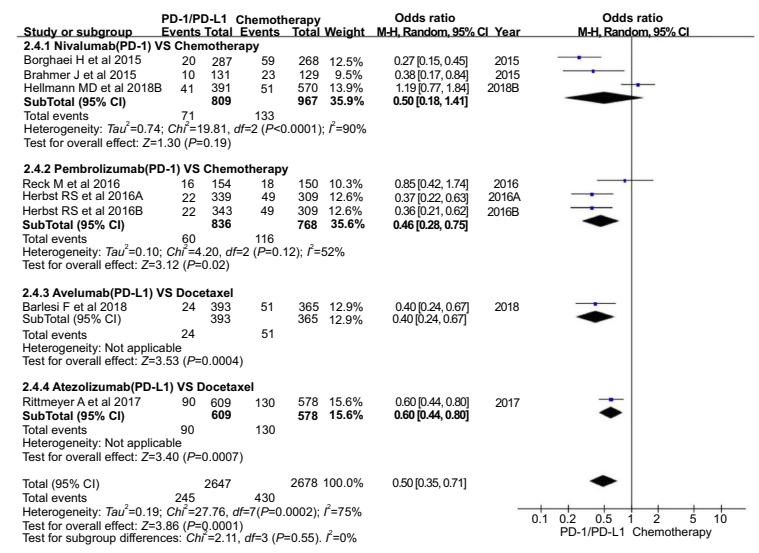

B

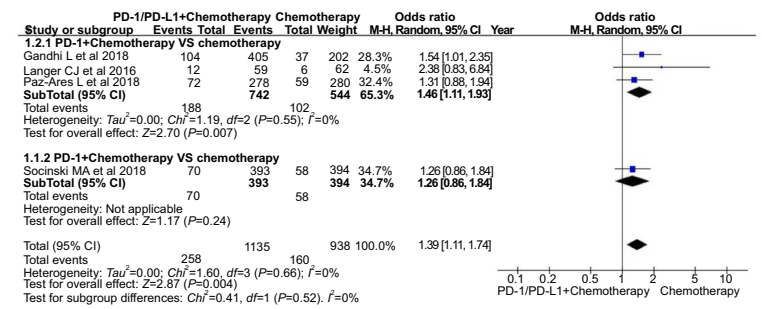

Figure 4 Forest plots of the incidence risk for grade I-2 diarrhea. (AI): OR of diarrhea for grade I-2 in the subgroup analysis (PD-I/PD-LI vs chemotherapy) (A2): OR of diarrhea for grade I-2 in the subgroup analysis (PD-I/PD-LI vs docetaxel/combined chemotherapy), the data included were assigned to the corresponding subgroup according to control group (docetaxel or combined chemotherapy). (A3): OR of diarrhea for grade I-2 in the subgroup analysis (PD-I/PD-LI vs chemotherapy), the data included were assigned to the corresponding subgroup according to the name of PD-I/PD-LI inhibitor and the control group. (B): OR of diarrhea for grade I-2 in the subgroup analysis (PD-I/PD-LI + chemotherapy VS chemotherapy).

Abbreviation: PD-I, programmed cell death I; PD-LI, programmed cell death ligand I; RE, random effect.

$\mathrm{Z}=1.11[p=0.27]$; Figure 5A1). ${ }^{13,18,20-22}$ Moreover, moderate heterogeneity results had emerged $\left(\mathrm{I}^{2}=46 \%\right)$, so the heterogeneity was considered to be derived from the two newly included data. ${ }^{13,18}$ When we performed a subgroup analysis based on the specific drug name of PD-1/PD-L1 inhibitor, no statistically significant analysis results could be found (Figure 5A3).

\section{Discussion}

Therapies that target the PD-1 receptor have shown unprecedented rates of durable clinical responses in patients with various cancer types. It was reported that PD-1 or PD-L1 inhibitor improved overall survival compared with standard-of-care chemotherapy in patients with metastatic NSCLC, and several of them have received regulatory approvals. ${ }^{29-31}$ With the development of clinical research, more and more PD-1/PD-L1 inhibitors have been tried in clinical trials for antilung cancer treatment and have shown good efficacy, ${ }^{8-10}$ especially for NSCLC. ${ }^{11-22}$ Similar to other antitumor drugs, good antitumor efficacy was along with many therapeutic side effects, especially for combination with chemotherapy.
Diarrhea is a common side effect of antitumor medications, such as docetaxel, irinotecan, and topotecan. Moderate or severe diarrhea can cause electrolyte imbalance in patients, further leading to the interruption of antitumor treatment. ${ }^{11-22}$ Docetaxel was used to be taken as a better choice for second-line treatment for advanced NSCLC based on improvement in survival versus best supportive care. ${ }^{32,33}$ Other treatment regimens, such as pemetrexed and erlotinib, did not show better efficacy than docetaxel in patients with NSCLC. ${ }^{34,35}$ In order to clarify the incidence of diarrhea in the treatment of NSCLC with PD-1/PD-L1 inhibitors, we conducted a systematic review and meta-analysis.

Through our meta-analysis of the included clinical trials, the incidence of diarrhea for all grades was significantly lower in the PD-1/PD-L1 inhibitor monotherapy group than in the chemotherapy group, while it was higher in PD-1/PD-L1 combined with chemotherapy compared with chemotherapy group alone (Figure 3). ${ }^{11-22}$ Similar incidence trend could also be seen in meta-analysis of diarrhea for grade 1-2 (Figure 4). ${ }^{11-16,18-22}$ Due to the existence of heterogeneity, we conducted a further subgroup analysis $\left(\mathrm{I}^{2}=79 \%\right)$, three different 

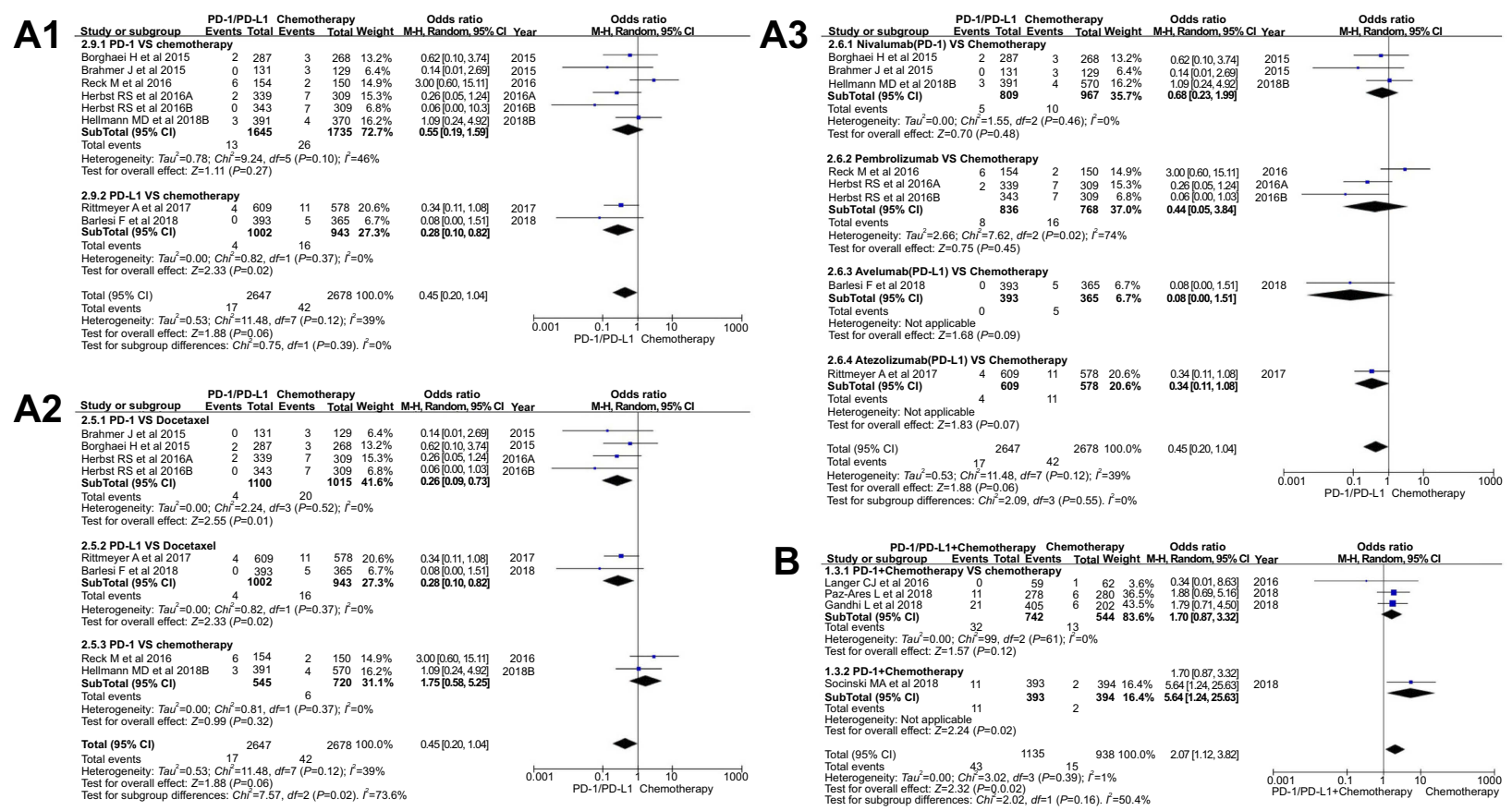

Figure 5 Forest plots of the incidence risk for grade 3-5 diarrhea. (AI): OR of diarrhea for grade 3-5 in the subgroup analysis (PD-I/PD-LI vs chemotherapy) (A2): OR of diarrhea for grade $3-5$ in the subgroup analysis (PD-I/PD-LI vs docetaxel/combined chemotherapy), the data included were assigned to the corresponding subgroup according to control group (docetaxel or combined chemotherapy). (A3): OR of diarrhea for grade 3-5 in the subgroup analysis (PD-I/PD-LI vs chemotherapy), the data included were assigned to the corresponding subgroup according to the name of PD-I/PD-LI inhibitor and the control group. (B): OR of diarrhea for grade 3-5 in the subgroup analysis (PD-I/PD-LI + chemotherapy vs chemotherapy).

Abbreviation: PD-I, programmed cell death I; PD-LI, programmed cell death ligand I; RE, random effect.

subgroup analysis methods were used for group A (Figure 3A1-A3). ${ }^{11,13,16-18,20-22}$ When we mixed the single-agent docetaxel and the combination chemotherapy together as a control group (Figures 3A1, 4A1 and 5A1), ${ }^{11,13,16-18,20-22}$ heterogeneity results emerged. So the heterogeneity was considered to be derived from the two newly included data (Reck et al, 2016 and Hellmann et al, 2018B). ${ }^{13,18}$

In order to eliminate the influence of heterogeneity on the analysis results, we conducted a comprehensive analysis of the results. Regardless of the degree of diarrhea (all grade, grade 1-2 and grade 3-5), the incidence risk of diarrhea in PD-1 monotherapy group was significantly lower than that of docetaxel monotherapy group, and the difference was of statistical significance. There is no obvious heterogeneity and publication bias among the included data (Figures 3A2, 4A2 and 5A2, Figures 13) ${ }^{20-22}$ When PD-1/PD-L1 inhibitor was combined with chemotherapy, the incidence risk of diarrhea was significantly increased. ${ }^{12,14,15,19}$

Although diarrhea was reported in $20 \%$ of the patients, some of grade 3-5 treatment-related diarrhea was reported..$^{8-22,36-39}$ They could be managed with treatment of the symptoms, and the patient recovered promptly without glucocorticoid treatment. ${ }^{36-39}$ Although diarrhea was reported as a common toxic side effect associated with PD-1/PD-L1 inhibitor appeared in clinical trials, no standard systematic treatment regimen for it was proposed. ${ }^{8-22,36-39}$ Therefore, for the fully understanding of PD-1/PD-L1-related diarrhea, more clinical trials and mechanism research are needed.

In a word, the incidence risk of diarrhea associated with single-agent PD-1/-PD-L1 inhibitors was significantly lower than that of the single-agent docetaxel group, while it was higher in PD-1/PD-L1 combined with chemotherapy group compared with chemotherapy group alone.

\section{Conclusions}

The incidence risk of diarrhea associated with single-agent PD-1/-PD-L1 inhibitors was significantly lower than that of docetaxel monotherapy group, while it was higher in PD-1/PD-L1 inhibitor combined with chemotherapy group compared with chemotherapy group.

\section{Statement of Ethics}

This study belongs to the type of data analysis and rearrangement and does not involve human- or animal-related ethical issues. 


\section{Abbreviation list}

PRISMA, Preferred Reporting Items for Systematic Reviews and Meta-Analyses; PD-1, programmed cell death-1; PD-L1, programmed cell death ligand 1; SCLC, small cell cancer; NSCLC, non-small cell lung cancer; RR, risk ratio; RE, random effect; FE, fixed effect.

\section{Acknowledgments}

This study was funded by the Natural Science Foundation of Shandong Province (ZR2015HL078), which were in charge by Yuan Tian.

\section{Author contributions}

All authors contributed to data analysis, drafting and revising the article, gave final approval of the version to be published, and agree to be accountable for all aspects of the work.

\section{Disclosure}

The authors have no conflicts of interest to report in relation to this paper.

\section{References}

1. Ribas A, Wolchok JD. Cancer immunotherapy using checkpoint blockade. Science. 2018;359(6382):1350-1355. doi:10.1126/science.aar4060

2. Tumeh PC, Harview CL, Yearley JH, et al. PD-1 blockade induces responses by inhibiting adaptive immune resistance. Nature. 2014;515(7528):568-571. doi:10.1038/nature13954

3. Waterhouse P, Penninger JM, Timms E, et al. Lymphoproliferative disorders with early lethality in mice deficient in Ctla-4. Science. 1995;270(5238):985-988.

4. Tivol EA, Borriello F, Schweitzer AN, Lynch WP, Bluestone JA, Sharpe AH. Loss of CTLA-4 leads to massive lymphoproliferation and fatal multiorgan tissue destruction, revealing a critical negative regulatory role of CTLA-4. Immunity. 1995;3(5):541-547.

5. Nishimura H, Nose M, Hiai H, Minato N, Honjo T. Development of lupus-like autoimmune diseases by disruption of the PD-1 gene encoding an ITIM motif-carrying immunoreceptor. Immunity. 1999;11(2):141-151.

6. Nishimura H, Okazaki T, Tanaka Y, et al. Autoimmune dilated cardiomyopathy in PD-1 receptor-deficient mice. Science. 2001;291 (5502):319-322. doi:10.1126/science.291.5502.319

7. Brahmer JR, Drake CG, Wollner I, et al. Phase I study of single-agent anti-programmed death-1 (MDX-1106) in refractory solid tumors: safety, clinical activity, pharmacodynamics, and immunologic correlates. $J$ Clin Oncol. 2010;28(19):3167-3175. doi:10.1200/JCO.2009.26.7609

8. Antonia SJ, Villegas A, Daniel D, et al. PACIFIC investigators. Overall survival with durvalumab after chemoradiotherapy in stage III NSCLC. N Engl J Med. 2018;379(24):2342-2350. doi:10.1056/NEJMoa1809697

9. Horn L, Mansfield AS, Szczęsna A, et al. IMpower133 study group. First-line atezolizumab plus chemotherapy in extensive-stage small-cell lung cancer. $N$ Engl J Med. 2018;379(23):2220-2229. doi:10.1056/NEJMoa1809064

10. Kowanetz M, Zou W, Gettinger SN, et al. Differential regulation of PD-L1 expression by immune and tumor cells in NSCLC and the response to treatment with atezolizumab (anti-PD-L1). Proc Natl Acad Sci USA. 2018;115(43):E10119-E10126. doi:10.1073/pnas.1802166115
11. Barlesi F, Vansteenkiste J, Spigel D, et al. Avelumab versus docetaxel in patients with platinum-treated advanced non-small-cell lung cancer (JAVELIN Lung 200): an open-label, randomised, phase 3 study. Lancet Oncol. 2018;19(11):1468-1479. doi:10.1016/S1470-2045(18)30673-9

12. Socinski MA, Jotte RM, Cappuzzo F, et al. IMpower150 study group. atezolizumab for first-line treatment of metastatic nonsquamous NSCLC. N Engl J Med. 2018;378(24):2288-2301. doi:10.1056/NEJMoa1716948

13. Hellmann MD, Ciuleanu TE, Pluzanski A, et al. Nivolumab plus ipilimumab in lung cancer with a high tumor mutational burden. $N$ Engl J Med. 2018;378(22):2093-2104. doi:10.1056/NEJMoa1801946

14. Paz-Ares L, Luft A, Vicente D, et al. KEYNOTE-407 investigators. pembrolizumab plus chemotherapy for squamous non-small-cell lung cancer. $N$ Engl J Med. 2018;379(21):2040-2051. doi:10.1056/ NEJMoa 1810865

15. Gandhi L, Rodríguez-Abreu D, Gadgeel S, et al. KEYNOTE-189 investigators. Pembrolizumab plus chemotherapy in metastatic non-small-cell lung cancer. $N$ Engl $J$ Med. 2018;378 (22):2078-2092. doi:10.1056/NEJMoa1801005

16. Rittmeyer A, Barlesi F, Waterkamp D, et al. Atezolizumab versus docetaxel in patients with previously treated non-small-cell lung cancer (OAK): a phase 3, open-label, multicentre randomised controlled trial. Lancet. 2017;389(10066):255-265. doi:10.1016/S01406736(16)32517-X

17. Fehrenbacher L, Spira A, Ballinger M, et al. Atezolizumab versus docetaxel for patients with previously treated non-small-cell lung cancer (POPLAR): a multicentre, open-label, phase 2 randomised controlled trial. Lancet. 2016;387(10030):1837-1846. doi:10.1016/ S0140-6736(16)00587-0

18. Reck M, Rodríguez-Abreu D, Robinson AG, et al. KEYNOTE-024 investigators. pembrolizumab versus chemotherapy for PD-L1positive non-small-cell lung cancer. $N$ Engl $J$ Med. 2016;375 (19):1823-1833. Epub 2016 Oct 8. doi:10.1056/NEJMoa1606774

19. Langer CJ, Gadgeel SM, Borghaei H, et al. KEYNOTE-021 investigators. Carboplatin and pemetrexed with or without pembrolizumab for advanced, non-squamous non-small-cell lung cancer: a randomised, phase 2 cohort of the open-label KEYNOTE-021 study. Lancet Oncol. 2016;17(11):1497-1508. doi:10.1016/S1470-2045(16)30498-3

20. Herbst RS, Baas P, Kim DW, et al. Pembrolizumab versus docetaxel for previously treated, PD-L1-positive, advanced non-small-cell lung cancer (KEYNOTE-010): a randomised controlled trial. Lancet. 2016;387(10027):1540-1550. doi:10.1016/S0140-6736(15)01281-7

21. Brahmer J, Reckamp KL, Baas P, et al. Nivolumab versus docetaxel in advanced squamous-cell non-small-cell lung cancer. $N$ Engl $J$ Med. 2015;373(2):123-135. doi:10.1056/NEJMoa1504627

22. Borghaei H, Paz-Ares L, Horn L, et al. Nivolumab versus docetaxel in advanced nonsquamous non-small-cell lung cancer. $N$ Engl J Med. 2015;373(17):1627-1639. doi:10.1056/NEJMoa1507643

23. Wang DY, Salem JE, Cohen JV, et al. Fatal toxic effects associated with immune checkpoint inhibitors: a systematic review and meta-analysis. JAMA Oncol. 2018;4(12):1721-1728. doi:10.1001/jamaoncol.2018.3923

24. Moher D, Liberati A, Tetzlaff J, Altman DG. PRISMA group preferred reporting items for systematic reviews and meta-analyses: the PRISMA statement. Ann Intern Med. 2009;151:264-69.W64. doi:10.7326/0003-4819-151-4-200908180-00135

25. Higgins JP, Altman DG, Gøtzsche PC, et al. Cochrane Statistical Methods Group. The Cochrane Collaboration's tool for assessing risk of bias in randomised trials. $B M J$. 2011;343:d5928.

26. Wells G, Shea B, O'Connell D, et al. The Newcastle-Ottawa Scale (NOS) for assessing the quality if nonrandomized studies in metaanalyses. 2009 http://www.ohri. ca/programs/clinical_epidemiology/ oxford.aspaccesse July 6, 2012. Doi:10.1094/PDIS-11-11-0999-PDN

27. Higgins JP, Thompson SG, Deeks JJ, Altman DG. Measuring inconsistency in meta-analyses. BMJ. 2003;327:557-560. doi:10.1136/ bmj.327.7420.895

28. DerSimonian R, Laird N. Meta-analysis in clinical trials. Control Clin Trials. 1986;7:177-188. doi:10.1016/0197-2456(86)90046-2 
29. Kazandjian D, Suzman DL, Blumenthal G, et al. FDA approval summary: nivolumab for the treatment of metastatic non-small cell lung cancer with progression on or after platinum-based chemotherapy. Oncologist. 2016;21:634-642. doi:10.1634/theoncologist.2015-0507

30. Sul J, Blumenthal GM, Jiang X, He K, Keegan P, Pazdur R. FDA approval summary: pembrolizumab for the treatment of patients with metastatic non-small cell lung cancer whose tumors express programmed death-ligand 1. Oncologist. 2016;21:643-650. doi:10.1634/theoncologist.2015-0498

31. Weinstock C, Khozin S, Suzman D, et al. US food and drug administration approval summary: atezolizumab for metastatic non-small cell lung cancer. Clin Cancer Res. 2017;23:4534-4539. doi:10.1158/ 1078-0432.CCR-17-0540

32. Fossella FV, DeVore R, Kerr RN, et al. Randomized phase III trial of docetaxel versus vinorelbine or ifosfamide in patients with advanced non-small-cell lung cancer previously treated with platinum-containing chemotherapy regimens. The TAX 320 non-small cell lung cancer study group. $J$ Clin Oncol. 2000;18:2354-2362. doi:10.1200/JCO.2000.18.10.2059

33. Shepherd FA, Dancey J, Ramlau R, et al. Prospective randomized trial of docetaxel versus best supportive care in patients with non-small-cell lung cancer previously treated with platinum-based chemotherapy. J Clin Oncol. 2000;18:2095-2103. doi:10.1200/ JCO.2000.18.10.2059

34. Hanna N, Shepherd FA, Fossella FV, et al. Randomized phase III trial of pemetrexed versus docetaxel in patients with non-small-cell lung cancer previously treated with chemotherapy. $J$ Clin Oncol. 2004;22:1589-1597. doi:10.1200/JCO.2004.08.040
35. Garassino MC, Martelli O, Broggini M, et al. Erlotinib versus docetaxel as second-line treatment of patients with advanced non-smallcell lung cancer and wild-type EGFR tumours (TAILOR): a randomised controlled trial. Lancet Oncol. 2013;14:981-988. doi:10.1016/S1470-2045(13)70310-3

36. Hamid O, Robert C, Daud A, et al. Safety and tumor responses with lambrolizumab (anti-PD-1) in melanoma. N Engl J Med. 2013;369 (2):134-144. doi:10.1056/NEJMoa1305133

37. Rizvi NA, Mazières J, Planchard D, et al. Activity and safety of nivolumab, an anti-PD-1 immune checkpoint inhibitor, for patients with advanced, refractory squamous non-small-cell lung cancer (CheckMate 063): a phase 2, single-arm trial. Lancet Oncol. 2015;16(3):257-265. doi:10.1016/S1470-2045 (15)70054-9

38. Hodi FS, Chesney J, Pavlick AC, et al. Combined nivolumab and ipilimumab versus ipilimumab alone in patients with advanced melanoma: 2-year overall survival outcomes in a multicentre, randomised, controlled, phase 2 trial. Lancet Oncol. 2016;17 (11):1558-1568. doi:10.1016/S1470-2045(16)30366-7

39. Migden MR, Rischin D, Schmults CD, et al. PD-1 blockade with cemiplimab in advanced cutaneous squamous-cell carcinoma. $N$ Engl J Med. 2018;379(4):341-351. doi:10.1056/NEJMoa1805131 


\section{Supplementary materials}

A1

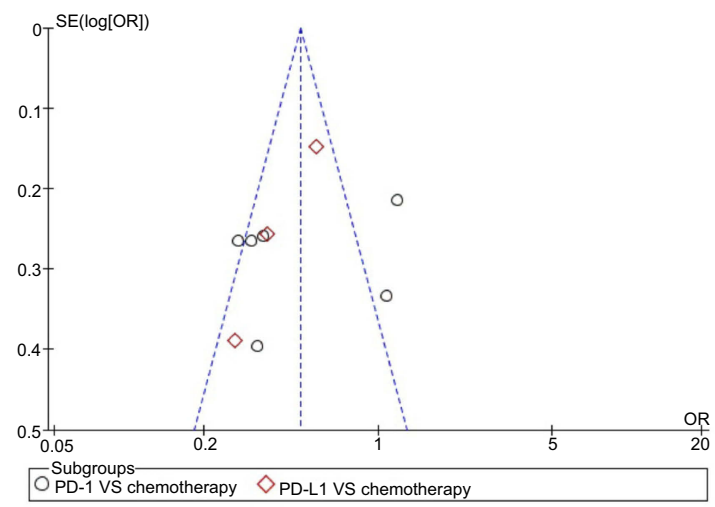

A2

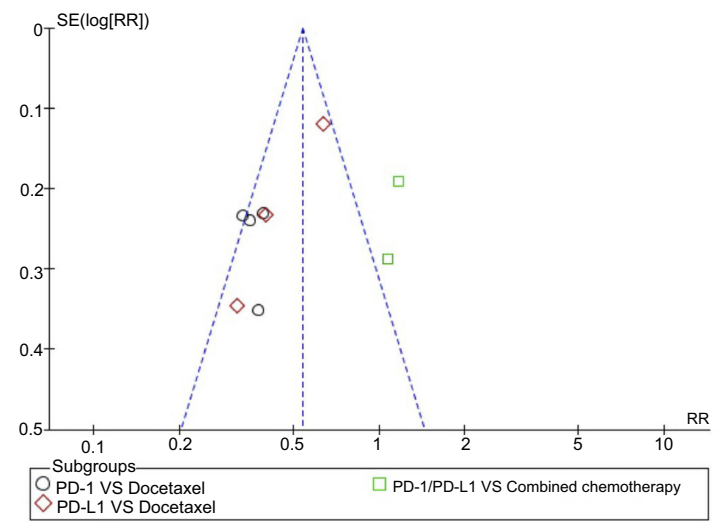

A3

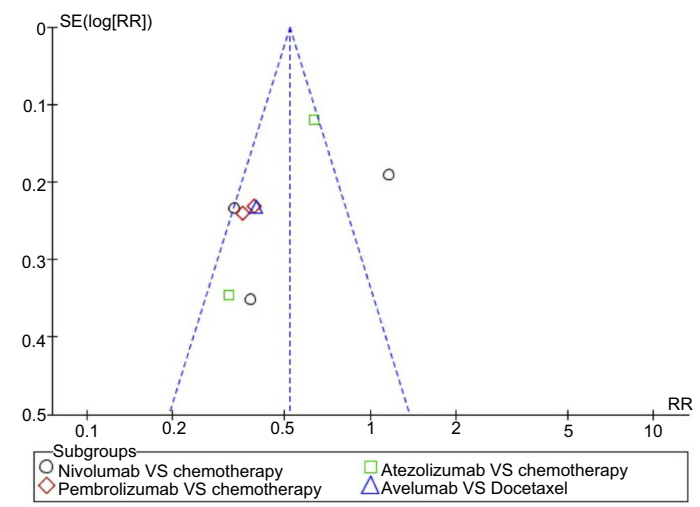

B

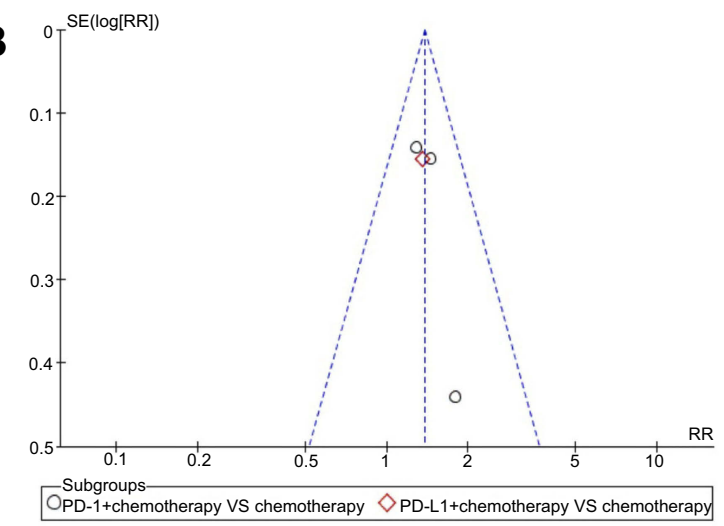

Figure SI Funnel plots of the incidence risk for all-grade diarrhea. (AI): Funnel plots of diarrhea for all grades in the subgroup analysis (PD-I/PD-LI vs chemotherapy). (A2): Funnel plots of diarrhea for all grades in the subgroup analysis (PD-I/PD-LI vs docetaxel/combined chemotherapy), the data included were assigned to the corresponding subgroup according to control group (docetaxel or combined chemotherapy). (A3): Funnel plots of diarrhea for all grades in the subgroup analysis (PD-I/ PD-LI vs chemotherapy), the data included were assigned to the corresponding subgroup according to the name of PD-I/PD-LI inhibitor and the control group. (B): Funnel plots of diarrhea for all grades in the subgroup analysis (PD-I/PD-LI + chemotherapy vs chemotherapy).

Abbreviation: PD-I, programmed cell death I; PD-LI, programmed cell death ligand I; OR, odds ratio; RR, risk ratio. 
A1

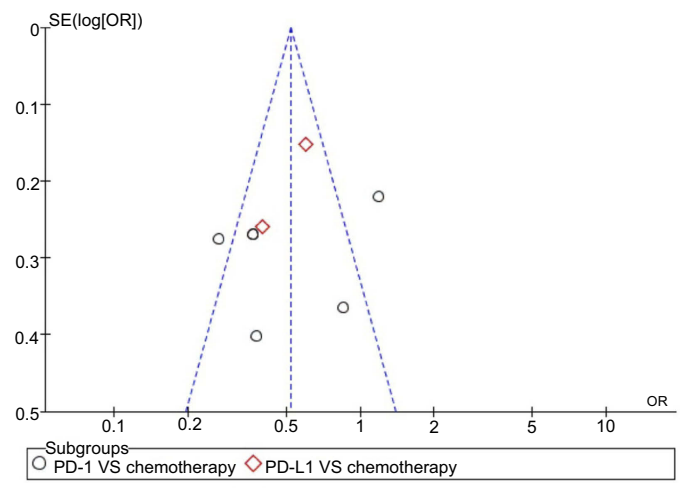

A2

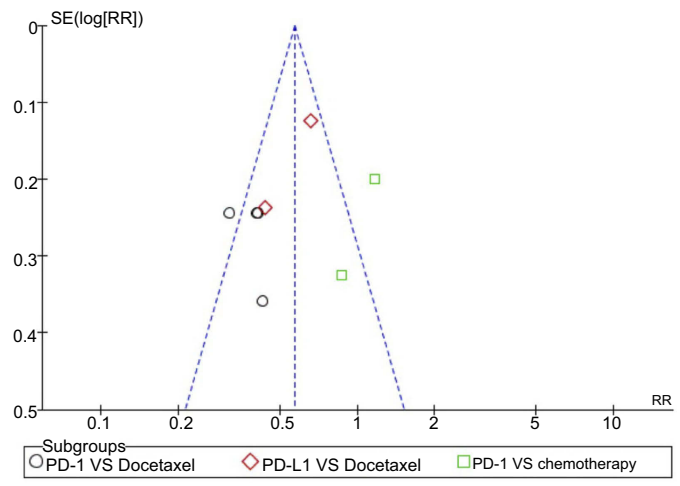

A3

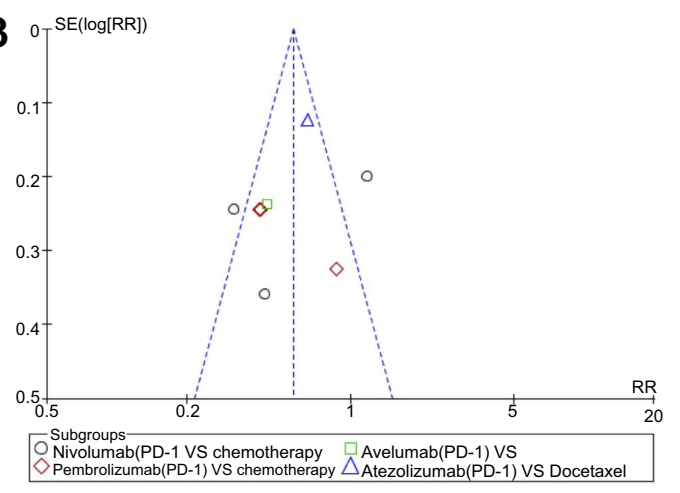

B

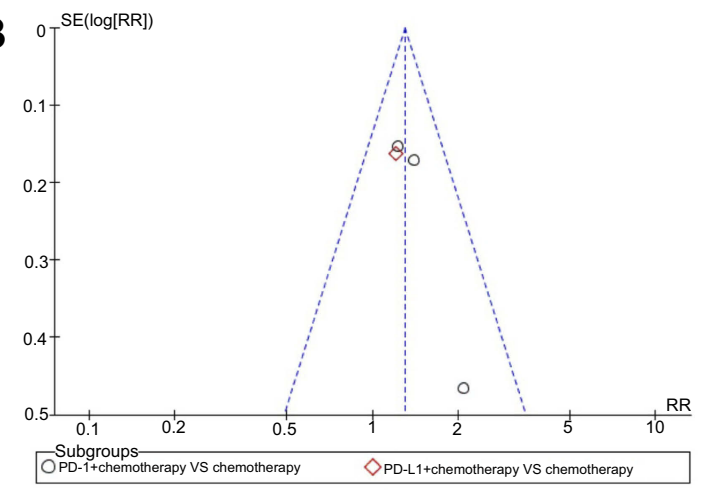

Figure S2 Funnel plots of the incidence risk for grade I-2 diarrhea. (AI): Funnel plots of diarrhea for grade I-2 in the subgroup analysis (PD-I/PD-LI vs chemotherapy). (A2): Funnel plots of diarrhea for grade I-2 in the subgroup analysis (PD-I/PD-LI vs docetaxel/combined chemotherapy), the data included were assigned to the corresponding subgroup according to control group (docetaxel or combined chemotherapy).(A3): Funnel plots of diarrhea for grade I-2 in the subgroup analysis (PD-I/PDLI vs chemotherapy), the data included were assigned to the corresponding subgroup according to the name of PD-I/PD-LI inhibitor and the control group. (B): Funnel plots of diarrhea for grade I-2 in the subgroup analysis (PD-I/PD-LI + chemotherapy vs chemotherapy).

Abbreviation: PD-I, programmed cell death I; PD-LI, programmed cell death ligand I; OR, odds ratio; RR, risk ratio. 
A1

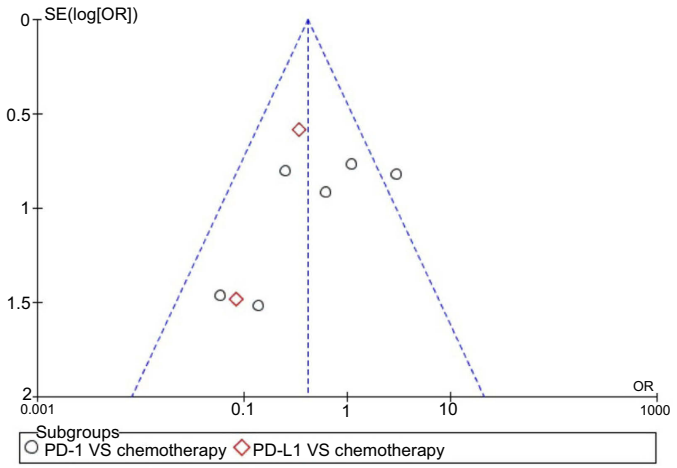

A2

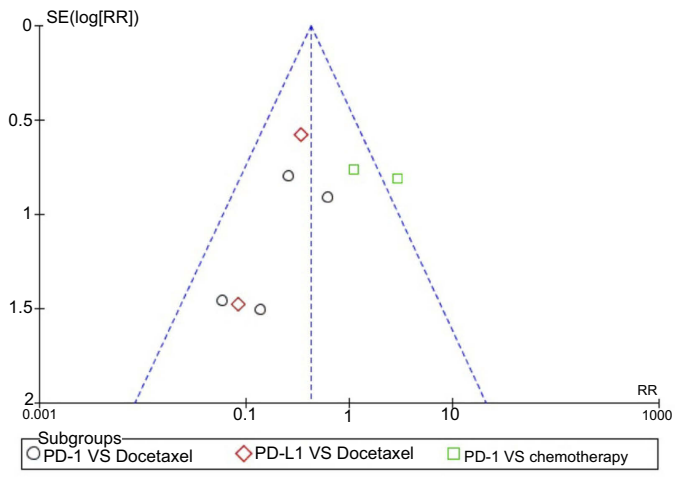

A3

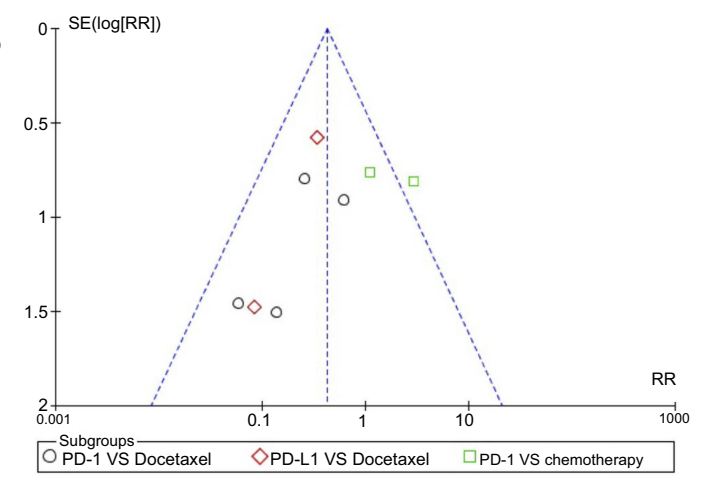

B

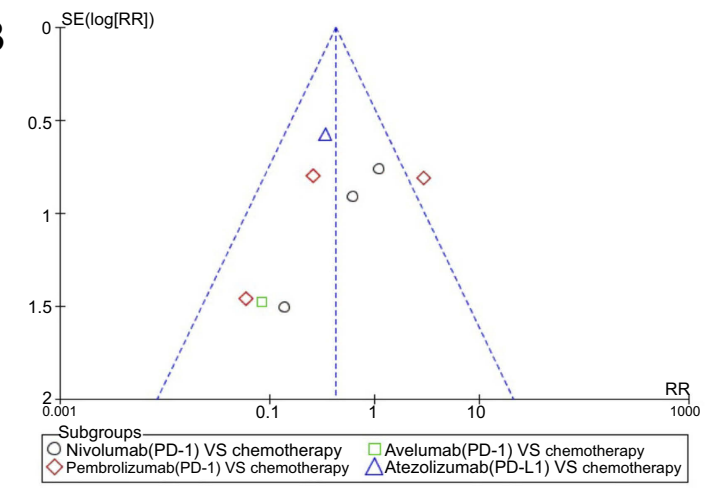

Figure S3 Funnel plots of the incidence risk for grade 3-5 diarrhea. (AI): Funnel plots of diarrhea for grade 3-5 in the subgroup analysis (PD-I/PD-LI vs chemotherapy). (A2): Funnel plots of diarrhea for grade $3-5$ in the subgroup analysis (PD-I/PD-LI vs docetaxel/combined chemotherapy), the data included were assigned to the corresponding subgroup according to control group (docetaxel or combined chemotherapy). (A3): Funnel plots of diarrhea for grade 3-5 in the subgroup analysis (PD- I/PDLI VS chemotherapy), the data included were assigned to the corresponding subgroup according to the name of PD-I/PD-LI inhibitor and the control group. (B): Funnel plots of diarrhea for grade 3-5 in the subgroup analysis (PD-I/PD-LI + chemotherapy vs chemotherapy).

Abbreviation: PD-I, programmed cell death I; PD-LI, programmed cell death ligand I; OR, odds ratio; RR, risk ratio.

\section{Publish your work in this journal}

Cancer Management and Research is an international, peer-reviewed open access journal focusing on cancer research and the optimal use of preventative and integrated treatment interventions to achieve improved outcomes, enhanced survival and quality of life for the cancer patient.
The manuscript management system is completely online and includes a very quick and fair peer-review system, which is all easy to use. Visit http://www.dovepress.com/testimonials.php to read real quotes from published authors. 